\title{
A Deep Learning Based Approach to Skin Lesion Border Extraction With a Novel Edge Detector in Dermoscopy Images
}

\author{
Abder-Rahman Ali ${ }^{1}$, Jingpeng $\mathrm{Li}^{1}$, Sally Jane O'Shea ${ }^{2}$, Guang Yang ${ }^{3}$, Thomas Trappenberg ${ }^{4}$, Xujiong Ye
}

\begin{abstract}
Lesion border detection is considered a crucial step in diagnosing skin cancer. However, performing such a task automatically is challenging due to the low contrast between the surrounding skin and lesion, ambiguous lesion borders, and the presence of artifacts such as hair. In this paper we propose a two-stage approach for skin lesion border detection: (i) segmenting the skin lesion dermoscopy image using U-Net, and (ii) extracting the edges from the segmented image using a novel approach we call FuzzEdge. The proposed approach is compared with another published skin lesion border detection approach, and the results show that our approach performs better in detecting the main borders of the lesion and is more robust to artifacts that might be present in the image. The approach is also compared with the manual border drawings of a dermatologist, resulting in an average Dice similarity of 87.7\%.
\end{abstract}

\section{INTRODUCTION}

The computerized analysis of skin cancer images normally starts by lesion border detection, which is considered a crucial step for subsequent diagnostic steps, given that clinical features such as border irregularity are measured from the border, an important feature (i.e. B feature) in the ABCD rule (criteria) that physicians, dermatologists and non-physicians could use to detect features of melanoma, enhancing its early diagnosis $[16,24]$. The $\mathrm{ABCD}$ acronym refers to the following four parameters: Asymmetry, Border irregularity, Color variegation, and Diameter greater than $6 \mathrm{~mm}$. Such parameters provide simple means for the appraisal of pigmented cutaneous lesions that may need to be further examined by a specialist with dermoscopy and excision where appropriate. Other clinical features such as atypical pigment network and globules rely on how well the border is detected. Detecting such borders automatically is however challenging due to the low contrast between the surrounding skin and the lesion, ambiguous lesion borders, artifacts such as hair, and the variation of colors inside the lesion [1].

Different methods have been proposed in literature for lesion border detection. Erkol et. al. [7] used Gradient Vector Flow (GVF) snakes to obtain the border of skin lesions in dermoscopy images, where a luminance image blurring

\footnotetext{
${ }^{1}$ A. Ali Faculty of Natural Sciences, Computing Science and Mathematics, University of Stirling, United Kingdom abder@cs.stir.ac.uk

${ }^{1} \mathrm{~J}$. Li Faculty of Natural Sciences, Computing Science and Mathematics, University of Stirling, United Kingdom jingpeng.Li@stir.ac.uk

${ }^{2}$ S. J. O'Shea Mater Private Hospital, Ireland sally.oshea@ucc.ie

${ }^{3}$ G. Yang National Heart Lung Institute, Imperial College London, United Kingdom g.yang@imperial.ac.uk

${ }^{4}$ T. Trappenberg Faculty of Computer Science, Dalhousie University, Canada tt@dal.cs.ca

${ }^{5} \mathrm{X}$. Ye School of Computer Science, University of Lincoln, United Kingdom xye@lincoln.ac.uk
}

approach is presented for automatic snake initialization. The approach is tested on 100 images (30 melanoma and 70 benign). The results show that 76 out of the 100 images have percentage border errors in less than $20 \%$, and 96 out of the 100 images have percentage border errors in less than $30 \%$. In [4] Celebi et. al. proposed a fast unsupervised approach to border detection of pigmented skin lesions based on the Statistical Region Merging (SRM) algorithm [5]. The authors attempted to facilitate the border detection process by the pre-processing operations of black frame removal and image smoothing. The process is also followed by a post-processing step, namely morphological dilation. The approach is tested on 90 dermoscopy images and does not perform well on images with a significant amount of hair. Celebi et.al. [2] proposed an unsupervised approach to border detection in dermoscopy skin lesion images based on a modified version of the JSEG algorithm [3] for segmentation, preceding that with pre-processing steps that aim at facilitating the border detection procedure (image smoothing, color quantization and approximate border localization), and carrying out a post-processing step as the final stage of the proposed approach. The approach is tested on 100 dermoscopy images (30 melanoma and 70 benign) and suffers from several limitations in that the bounding box determined by the approximate lesion localization method does not contain the whole lesion, and the method might not perform well on images with a significant amount of hair. An automatic approach for skin lesion border detection was proposed by Tzekis et. al. [6] where the process is carried out through a combination of different phases. The first phase attempts to determine if some points belong to melanoma or not, which is carried out by calculating the base color between the skin color and the melanoma color. The second phase of the approach locates a random point in the mole either from the entire area of the image or from random points around the center of the image. A point on the border of the mole is then found in the third phase, which adds more border points and terminates when a point is very close to where the first one is located, eventually drawing the actual boundaries of the lesion. The results of the approach have been shown to be fast, simple and accurate. The authors also utilized an algorithm for hair removal. An Artifact Removal and Border Detection (ARBD) approach was proposed by Abbas [8]. The approach is composed of multiple steps: preprocessing to enhance the contrast of lesions, artifact removal to reduce the effects of specular reflection, dermoscopic-gel, and lines (e.g. hair, blood vessels, skin lines, ruler markings and camera flash), plane-fitting to reduce the effect of texture 
patterns from tumor regions, detecting candidate regions, and segmenting the tumor region by Adaptive Dynamic Programming (ADP). The approach is applied on 250 dermoscopic imagines (30 benign melanocytic, 60 Malignant Melanomas (MM), 45 Basal Cell Carcinoma (BCC), 25 Merkel Cell Carcinoma (MCC), 70 Seborrhoeic Keratosis (SK), and 20 Acral volar Melanocytic (AM)), and has shown to be robust to boundary detection of the tumor in cases of fuzzy or smooth lesion types (BCC, MCC and AM). Other studies attempting to detect skin lesion borders can be found in [2629], and a comprehensive survey on lesion border detection in dermoscopy images can be found in [9].

With the increasing interests in Deep Learning in recent years, along with the outstanding results it has provided on different computer vision tasks, some attempts have been made in utilizing this technology in skin lesion border detection. Sabouri and GholamHosseini [10] used a Convolutional Neural Network (CNN) to detect lesion borders. The CNN is trained on two classes: the lesion class (containing 480 lesion images) and the background class (containing 1200 images of the normal skin with artifacts such as hair and ruler marker). The output is a binary mask labeling the segmented lesion. Lesion borders using the CNN are not accurately detected. The authors thus utilized the morphological closing operation as a post-processing step to determine the border more accurately. The advantage of this approach is that no pre-processing operations are carried out for hair removal and illumination correction. In [11] the authors employed a $\mathrm{CNN}$ as feature extractor avoiding any pre-processing for the input images, and a Support Vector Machine (SVM) as a skin lesion classifier. The border of the lesion is obtained through combining the binary mask with the original image. The CNN is trained on 23,000 patches of normal and skin lesions divided in such a way that each class contains the same number of images. Results show that some lesion borders are not detected perfectly, and thus a post-processing approach of smoothing the images is applied.

In this paper, we propose a skin lesion border detection method that is composed of two stages: (i) segmenting the skin lesion dermoscopy image using U-Net, and (ii) detecting the edge (border) using a novel method called FuzzEdge. As opposed to most previous studies, our approach requires neither pre-processing (i.e. image enhancement) nor postprocessing (i.e. mathematical morphology) steps. Section (II) explains the U-Net architecture, Section (III) explains our novel fuzzy border detector, Section (IV) provides an overview and explanation of results, and the paper is concluded in Section (V).

\section{IMAGE SEGMENTATION USING CONVOLUTIONAL NEURAL NETWORKS}

CNNs tend to produce dense predictions by extracting patches around each pixel which can be inefficient. A Fully Convolutional Network (FCN) is proposed in [13-14], which uses up-sampling and fully convolutional layers to generate pixel-wise predictions in a more efficient manner in a single pass [12]. In this paper, we use the U-Net architecture

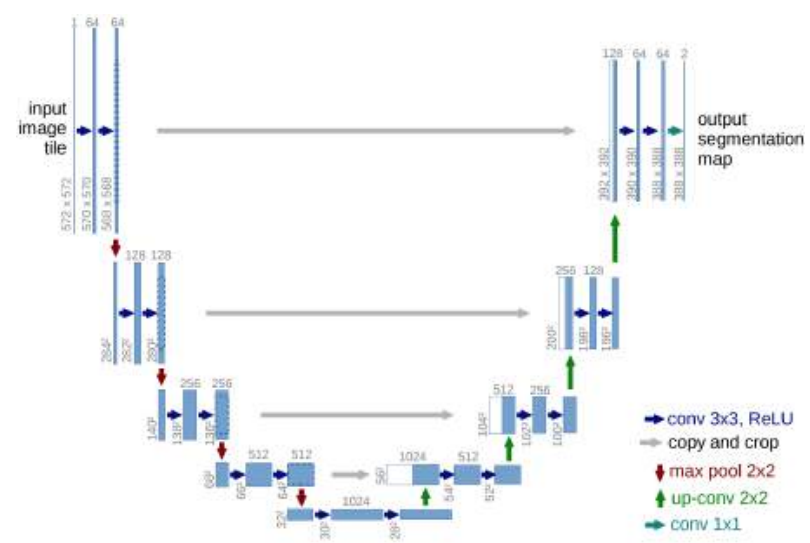

Fig. 1: U-Net architecture: blue boxes correspond to a multichanel feature map with the number of channels shown on top of the box. The value on the lower left edge of the box is the $x$-y-size. The copied feature maps are shown in white boxes, and the arrows refer to different operations [15]

[15] for segmenting the mole images. U-Net is an endto-end encoder-decoder network for semantic segmentation which was firstly used for medical image segmentation. The architecture is composed of left (down) and right (up) sides. The down part, which follows the typical convolutional network architecture, is the encoder part where convolution blocks are applied followed by maxpooling in order to encode the input image into feature representations at multiple levels. The number of feature channels are doubled at each downsampling step. On the other hand, the up part consists of upsampling the feature map followed by a convolution operation that brings the number of feature channels to half; a concatenation with the corresponding cropped feature map from the down part occurs, followed by two $3 \times 3$ convolutions which are also followed by two ReLU operations and one $2 \times 2$ max-pooling operation with stride 2 used for downsampling. The cropping process is essential as border pixels are lost at each convolution. Thus, while upsampling we concatenate the higher resolution features from the down part with the upsampled features in order to localize and learn representations better. The resulting architecture is that the expansive path is symmetric to the concatenating path, yielding a u-shaped architecture (Fig. 1). The final layer of U-Net uses a $1 \times 1$ convolution to map each 64 feature vector to the desired number of classes. The network is composed of 23 convolutional layers in total, provided that it does not have any fully connected layers and uses only the valid part of each convolution. For the border region of the image, the pixels are predicted by an overlaptile strategy such that the missing context is extrapolated by mirroring the input image, thus allowing the U-Net network to be applied on large images. The outcome of this stage is the segmented image, where the foreground (in white) represents the skin lesion and the background (in black) represents the skin. 


\section{FUZZY BORDER DETECTOR}

Based on the work in [25], in this paper we propose a novel fuzzy filter for skin lesion border detection, namely FuzzEdge. The major adaptation is that the fuzzy filter proposed in [25] calculates the weighted fuzzy mean for the purpose of noise removal, while our FuzzEdge calculates the standard deviation for the purpose of edge detection. The algorithm code has been open sourced and can be accessed via https://github.com/abderhasan/fuzzedge.

Assume we have an image $G$ of size $M \times N$ pixels, and has $L$ gray levels. Such image can be denoted as $G=$ $[g(i, j)]_{M \times N}$, where $g(i, j) \in\{0,1, \ldots, L-1\}$ refers to a pixel in the image. Fuzzy sets that represent a particular concept for the gray level (i.e. intensity feature) of the image pixels can be constructed. Examples of such fuzzy sets are dark pixels and bright pixels. The membership function of each fuzzy set determines the membership grade in the range $[0,1]$ (1: full membership, 0: no membership) by which a pixel with a certain gray level belongs to that fuzzy set (concept). Those fuzzy sets can be eventually used to describe image pixels.

The fuzzy concepts can be derived from the image histogram, with gray levels in the range $[0, L-1]$ defined as follows:

$$
h\left(g_{k}\right)=\frac{n_{k}}{n}
$$

where $g_{k}$ denotes the $k^{\text {th }}$ gray level of image $G, n_{k}$ is the number of pixels with the $k^{\text {th }}$ gray level in $G, n$ is the total number of pixels in $G$, and $k=0,1,2, \ldots, L-1$.

In order to generate the fuzzy sets (concepts), the membership functions for those fuzzy concepts have to first be defined. A heuristic algorithm is utilized to define the membership functions of the different fuzzy concepts from the histogram of the image. Three fuzzy concepts that represent an image have been created: Bright, Dark, and Median.

The fuzzy concepts used in this work are of the $L-R$ type fuzzy number [23] defined as follows:

$$
f_{L R_{-} F C}(x)= \begin{cases}L\left(\frac{m-x}{\alpha}\right), & x \leq m \\ R\left(\frac{x-m}{\beta}\right), & x \geq m\end{cases}
$$

where $L(y)=R(y)=\max (0,1-y)$, and $f(x)$ can be represented as a triplet $[m, \alpha, \beta]$, such that $m$ corresponds to the modal value of the membership function, and $\alpha$ and $\beta$ are the spreads that correspond to the left-hand and right-hand curves of the membership function, respectively. Algorithm 1 highlights the steps required to create fuzzy sets.

For initiating the filtering process, a $3 \times 3$ window (kernel) is affected on the input image, where the window determines the gray level values (intensities) of the filtered area, and the pixel to be filtered would stand in the central cell of the $3 \times 3$ kernel. Let $X=[x(i, j)]_{M \times N}$ be the original input image, and $Y=[y(i, j)]_{M \times N}$ be the filtered output image. The $(i, j)^{t h}$ pixel of the filtered image $Y$ is represented input : grayscale image I
output fuzzy set (concept)

:

1 For the fuzzy concepts Bright, Dark, and Median, specify the intervals of [Bright $t_{\text {begin }}$, Bright $\left._{\text {end }}\right]$, [Dark begin $_{\text {,Dark }}$ end], and

[Median begin $_{\text {, Median }}$ end], respectively.

2 Let Bright $_{\text {begin }}=\left(N_{f}-1\right)\left[\frac{L-1}{N_{f}}\right]$, Dark $_{\text {end }}=\left[\frac{L-1}{N_{f}}\right]$, Median $_{\text {begin }}=$ Dark $_{\text {end }}-$ left_overlap, and Median $_{\text {end }}=$ Bright $_{\text {begin }}+$ right_overlap $/ * N_{f}$ is the number of fuzzy concepts, and left_overlap and right_overlap determine the overlapping range of the fuzzy concepts (the overlap range was set to 0 in this paper) $*$.

3 Set Dark ${ }_{\text {begin }}$ to be the first $g_{k}$ from 0 to Dark ${ }_{\text {end }}$.

4 Set Bright end to be the last $g_{k}$ from Bright begin to $L-1$.

5 In interval [Dark begin $_{\text {, }}$ Dark $_{\text {end }}$ ], find a pixel $g_{k}$ with the maximum value of $p\left(g_{k}\right)$.

6 For the fuzzy concept Dark, create its membership function $f_{\text {Dark }}$ as follows: $m_{\text {Dark }} \leftarrow g_{k}$,

$\alpha_{\text {Dark }} \leftarrow m_{\text {Dark }}-$ Dark $_{\text {begin }}$,

$\beta_{\text {Dark }} \leftarrow$ Dark $_{\text {end }}-m_{\text {Dark }}$.

7 In interval [Median ${ }_{\text {begin }}$, Median $\left._{\text {end }}\right]$, find a pixel $g_{k}$ with the maximum value of $p\left(g_{k}\right)$.

8 For the fuzzy concept Median, create its membership function $f_{\text {Median }}$ as follows: $m_{\text {Median }} \leftarrow g_{k}$, $\alpha_{\text {Median }} \leftarrow m_{\text {Median }}-$ Median $_{\text {begin }}$, $\beta_{\text {Median }} \leftarrow$ Median $_{\text {end }}-m_{\text {Median }}$.

9 In interval [Bright, begin-Bright $\left.{ }_{\text {end }}\right]$, find a pixel $g_{k}$ with the maximum value of $p\left(g_{k}\right)$.

10 For the fuzzy concept Bright, create its membership function $f_{\text {Bright }}$ as follows: $m_{\text {Bright }} \leftarrow g_{k}$,

$\alpha_{\text {Bright }} \leftarrow m_{\text {Bright }}-$ Bright $_{\text {begin }}$,

$\beta_{\text {Bright }} \leftarrow$ Bright $_{\text {end }}-m_{\text {Bright }}$.

Algorithm 1: Fuzzy set (concept) creation process

as: $y(i, j)=F u z z E d g e(\underline{X}(i, j))$, where $\underline{X}(i, j)$ is a $3 \times 3$ kernel centered at the input pixel $x(i, j)$ that will be affected by the filter, and FuzzEdge(.) denotes the function of the FuzzEdge (fuzzy filter). The kernel can be represented as follows:

$$
\underline{X}(i, j)=\left[\begin{array}{ccc}
x(i-1, j-1) & x(i-1, j) & x(i-1, j+1) \\
x(i, j-1) & x(i . j) & x(i, j+1) \\
x(i+1, j-1) & x(i+1, j) & x(i+1, j+1)
\end{array}\right]
$$

(3)

The FuzzEdge operation is composed of three standard deviation processes, one for each fuzzy concept (Bright, Dark, or Median). Each standard deviation process determines the value of the filtered pixel on one of the three fuzzy concepts (fuzzy intensity features). The weight associated with each pixel is determined by referring to the membership function (i.e. $f_{\text {Bright }}$ ) of the associated fuzzy concept. The standard deviation is then found for the pixels located in the 
kernel, eventually affecting the result to the pixel located at the center of the kernel (i.e. $\bar{y}(i, j))$. This process allows us to find $y_{B \overline{r i g h t}}(i, j), y_{\overline{D a r k}}(i, j)$, and $y_{M \bar{e}}{ }_{\text {dian }}(i, j)$.

After $y_{B r i g h t}(i, j), y_{\text {Dark }}^{-}(i, j)$ and $y_{M e \overline{d i a n}}(i, j)$ are produced, the decision process of FuzzEdge is utilized in order to determine the final filtered output of each pixel in the input image by referring to a fuzzy estimator derived from a fuzzy interval. A fuzzy interval is of $L R-t y p e$ if two shape functions $L$ and $R$ exist, in addition to the parameters (i.e. $\left(m_{l}, m_{R}\right) \in R^{2}, \alpha$ and $\beta$ ) that are used to form the membership function of the fuzzy interval. The fuzzy interval can be denoted as $F I=\left[m_{l}, m_{r}, \alpha, \beta\right]_{L R}$, and the membership function of $F I$ can be defined as shown in Equation 4. A standard deviation process similar to the one described above is applied on a sliding kernel (window) centered at the input pixel $x(i, j)$. In the fuzzy estimator step, the parameters are assigned the following values: $m_{r}=B$ Bight $t_{\text {end }}, m_{l}=$ Dark $_{\text {begin }}, L(y)=0$ and $R(y)=0$ for the membership function of the fuzzy interval.

$$
f_{L R_{-} F I}(x)= \begin{cases}L\left(\frac{m_{l}-x}{\alpha}\right), & x \leq m_{l} \\ 1, & m_{l} \leq x \leq m_{r} \\ R\left(\frac{x-m_{r}}{\beta}\right), & x \geq m_{r}\end{cases}
$$

The final output of each filtered pixel is determined by selecting the pixel that is nearest to the fuzzy estimator from $y_{\text {Bright }}(i, j), y_{\overline{\text { Dark }}}^{-}(i, j)$ and $y_{M \bar{e}}{ }_{\text {dian }}(i, j)$ (see Algorithm 2).

$$
\begin{array}{l|l}
1 & \text { if }\left|\bar{y}_{\text {Dark }}(i, j)-f_{L R-F I}(\underline{X}(i, j))\right|< \\
2 & \left|\bar{y}_{\text {Median }}(i, j)-f_{L R-F I}(\underline{X}(i, j))\right| \\
2 & y(i, j) \leftarrow \bar{y}_{\text {Dark }}(i, j) \\
3 & \text { else } \\
4 & y(i, j) \leftarrow \bar{y}_{\text {Median }}(i, j) \\
5 & \text { if }\left|\bar{y}_{\text {Bright }}(i, j)-f_{L R-F I}(\underline{X}(i, j))\right|< \\
6 & \left|y(i, j)-f_{\text {LR-FI }}(\underline{X}(i, j))\right| \\
6 & y(i, j) \leftarrow \bar{y}_{\text {Bright }}(i, j)
\end{array}
$$

\section{Algorithm 2: Decision process of FuzzEdge}

The general framework of the proposed approach can be shown in Fig. 2.
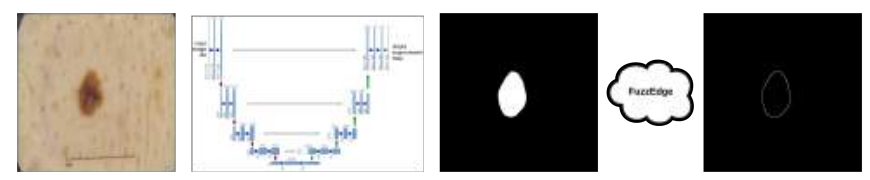

Fig. 2: General framework

\section{RESULTS AND DISCUSSION}

The U-Net architecture is trained on 2594 dermoscopy images along with their corresponding ground truth response masks from the "ISIC 2018: Skin Lesion Analysis Towards Melanoma Detection" grand challenge datasets [18-19]. Fig.
3 depicts some examples on the training dataset and the corresponding ground truth.

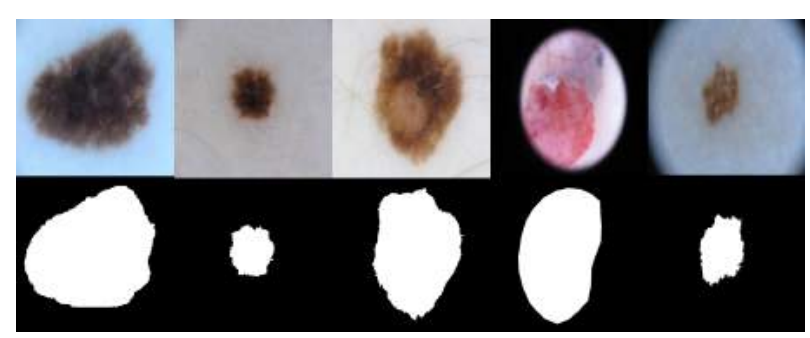

Fig. 3: Training examples

To make the most of the training data, augmentation using some transformations has been applied (such as rotation, shifting horizontally and vertically, shearing, zooming, horizontal flip, and filling in newly created pixels which can appear after a rotation or a horizontal/vertical shift). This avoids the model from coming across the exact same image twice, and thus preventing over-fitting and leading to better generalization. Images used have been resized to $512 \times 512$. U-Net was implemented using Keras and FuzzEdge using Python. The U-Net model is trained for 20 epochs on a Tesla P100 GPU. Fig. 4 shows how accuracy is improved across the epochs.

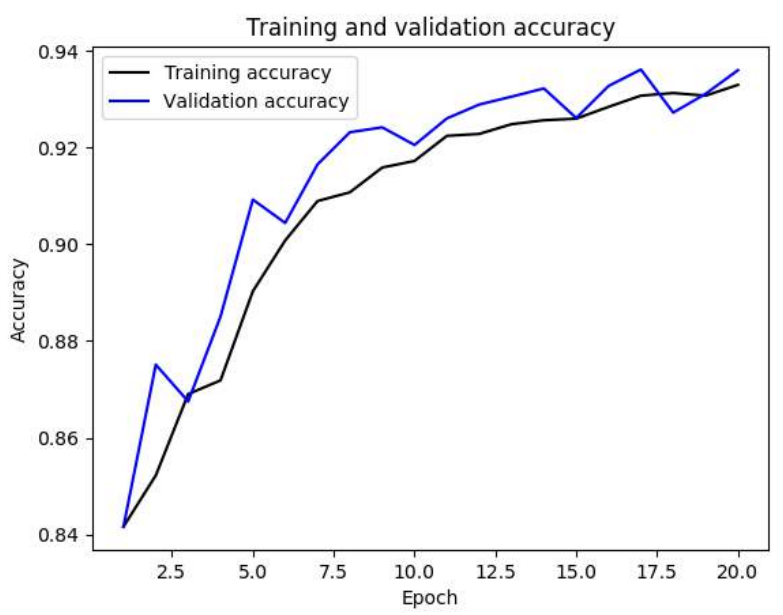

Fig. 4: Accuracy improvement across different epochs

\section{A. Ground truth}

A ground truth is required to compare our automatically detected borders against. The manual borders were created by a dermatologist (Dr.Sally Jane O'Shea). Fig. 5 shows an example on such annotated images. Those images represent a sample of skin lesion dermoscopy test images that the model has not been trained on before (i.e. did not see). 


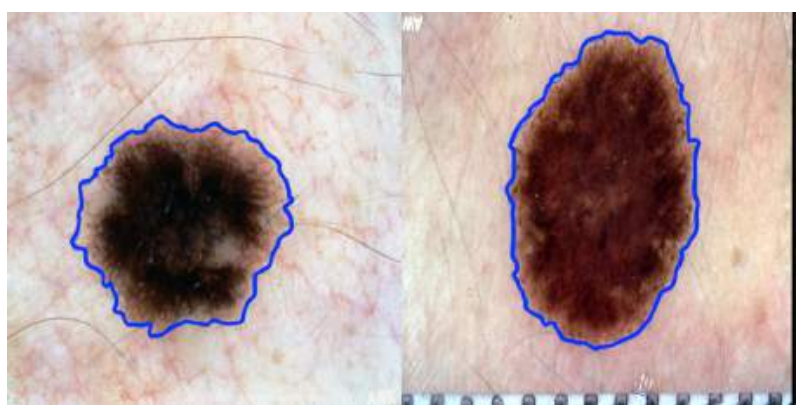

Fig. 5: Borders (in blue) manually drawn by a dermatologist

\section{B. Comparison with another automated method}

Fig.6 shows the test images, segmentation results (i.e. U-Net output) and borders detected (i.e. FuzzEdge output).
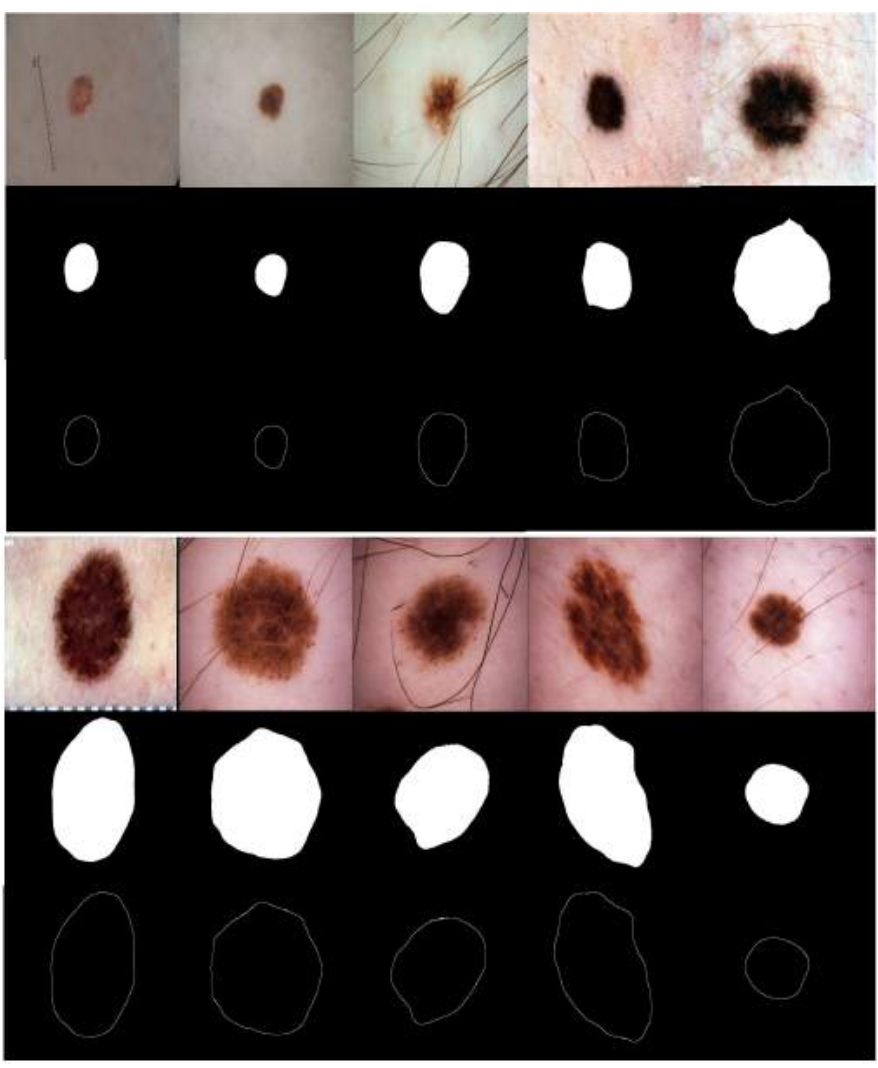

Fig. 6: Results of our proposed approach. The first row in the two sets of images represents the original dermoscopy images, the second row shows the segmentation results of U-Net, and the third row displays the detected borders after applying FuzzEdge.

We compare our approach with another automatic border detection approach proposed by Hua [21]. Fig.7 illustrates Hua's two stage results: (i) pre-processing and (ii) edge detection using the Sobel edge detector [22] (the author utilizes different edge detectors, but we chose one edge detector for brevity).
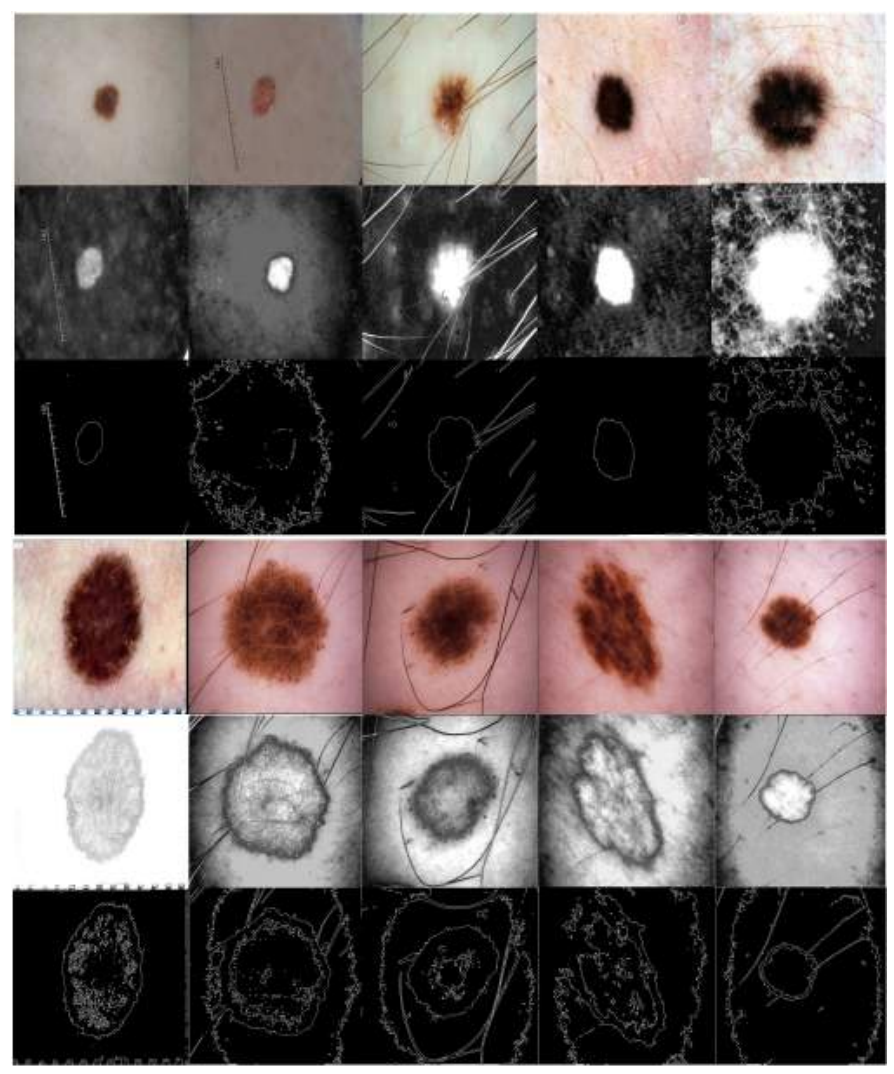

Fig. 7: Results of the approach proposed by Hua [21]. The first row in the two sets of images represents the original dermoscopy images, the second row shows pre-processing results, and the third row displays the detected borders after applying the Sobel edge detector.

From Fig. 6 and Fig. 7 we can notice that the proposed approach is able to detect the main border around the skin lesion, and is robust to artifacts presented in the images as opposed to the approach proposed by Hua [21]. Moreover, the approach by Hua is more of a heuristic approach on a trial and error basis as different threshold and sigma values need to be experimented with. However, our proposed approach lacks the ability to detect the exact fine structure of the skin lesion border. This might be due to the many structures that skin lesions possess, unlike other medical imaging shapes (i.e. colon) that tend to be very similar, This might also be due to downsampling the images in the U-Net process.

\section{Comparison with the ground truth}

Fig. 8 shows a sample of our results (white border) overlaid against the dermatologist's annotations (blue border). A visual comparison between the borders drawn by the dermatologist and the borders of our proposed approach shows the automatically extracted borders are very close to those manually outlined by the dermatologist. 


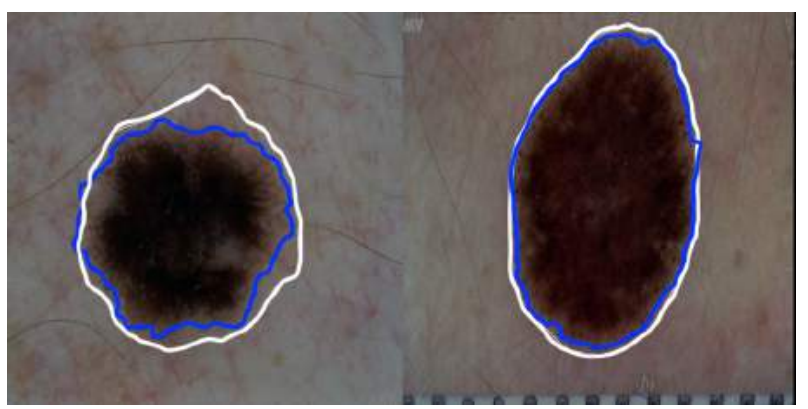

Fig. 8: Borders of our proposed approach (in white) overlaid on the borders by the dermatologist (in blue)

To quantitatively evaluate our method against the ground truth, we fill the area surrounded by the dermatologist's border and our method's border for each image. We then use the Dice coefficient [31] to measure the similarity between the two images. The Dice coefficient is a measure that finds the spatial overlap between two binary images, resulting in a value that lies between 0 (no overlap) and 1 (agree perfectly). The Dice coefficient can be defined as follows:

$$
D=\frac{2(A \cap G)}{A+G} \times 100 \%
$$

where $A$ is the algorithm output and $G$ is the ground truth.

Table. 1 lists the Dice coefficient results of 10 test images (the names reflect the original names as used in the dataset). The Dice similarity achieved by our approach is $87.7 \%$ on average.

TABLE I: Dice values for dermoscopy test images

\begin{tabular}{cc}
\hline Image & Dice \\
\hline ISIC_0012456 & $82.6 \%$ \\
ISIC_0012560 & $89 \%$ \\
ISIC_0012647 & $81.1 \%$ \\
ISIC_0015094 & $84.7 \%$ \\
ISIC_0015117 & $88.2 \%$ \\
ISIC_0015165 & $95.4 \%$ \\
ISIC_0017789 & $74.8 \%$ \\
ISIC_0018917 & $94.4 \%$ \\
ISIC_0019133 & $94.5 \%$ \\
ISIC_0020005 & $92.1 \%$ \\
\hline
\end{tabular}

\section{CONCLUSION}

In this paper we propose a deep learning based approach for skin lesion border detection in dermoscopy images. Compared to another automatic approach, our approach proves to be more robust to artifacts that might be present in skin lesion images (i.e. hair). When compared to a dermatologist's manual border drawing (i.e. ground truth), our approach resulted in an average Dice similarity of $87.7 \%$. An important feature of the proposed approach lies at that no pre-processing (e.g. image enhancement) or post-processing (e.g. mathematical morphology) methods are required. However, our approach fails to detect the exact structure of the skin lesion border. This might be mainly due to the many skin lesion shapes, which require more data when training the U-Net. As the future work, we plan to investigate further how to capture the fine structures of the skin lesion borders as this is a crucial factor in determining the border irregularity.

\section{REFERENCES}

[1] M. Celebi, H. Kingravi, H. Iyatomi, Y. Aslandogan, W. Stoecker, R. Moss, et al, "Border detection in dermoscopy images using statistical region merging," Skin Research and Technology, 14(3), 347-353, 2008.

[2] M. Celebi, Y. Aslandogan, W. Stoecker, H. Iyatomi, H. Oka, and X. Chen, "Unsupervised border detection in dermoscopy images," Skin Res. Technol., vol. 13, no. 4, pp. 454-462, 2007.

[3] Y. Deng, BS. Manjunath, "Unsupervised segmentation of color-texture regions in images and video," IEEE Trans Pattern Analysis Machine Intelligence, 23:800-810, 2001.

[4] M. Celebi, H. Kingravi, and J. Lee, "Fast and accurate border detection in dermoscopy images using statistical region merging," in Proc. SPIE Medical Imaging, 2007.

[5] R. Nock and F. Nielsen, "Statistical Region Merging," IEEE Trans. on Pattern Analysis and Machine Intelligence, 26(11): 1452-1458, 2004.

[6] P. Tzekis, A. Papastergiou, A. Hatzigaidas, Z. Zaharis, D. Kampitaki, P. Lazaridis, M. Goula, "A simple algorithm for automated skin lesion border detection," WSEAS Transactions on Signal Processing, vol.5, issue 6, pp. 229-240, 2009

[7] B. Erkol, R. H. Moss, R. J. Stanley, W. V. Stoecker, and E. Hva-tum, "Automatic lesion boundary detection in dermoscopy images using gradient vector flow snakes," Skin Res. and Technol., vol. 11, pp. 1726, 2005.

[8] Q. Abbas, "A aoftware approach for border detection using pigmented skin lesions," IJCSNS International Journal of Computer Science and Network Security, vol.17, no.5, 2017.

[9] M. Celebi, Q. Wen, H. Iyatomi, K. Shimizu, H. Zhou and G. Schaefer, "A state-of-the-art survey on lesion border detection in dermoscopy images," in Dermoscopy Image Analysis, M. E. Celebi, T. Mendonca and J. S. Marques, Eds., Boca Raton, CRC Press, pp. 97-129, 2015.

[10] P. Sabouri and H. GholamHosseini, "Lesion border detection using deep learning," in Proceedings of the 2016 IEEE Congress on Evolutionary Computation (CEC), Vancouver, BC, Canada, pp.1416-1421, 2016.

[11] A. Jadhav, A. Ghontale, V. Shrivastava, "Segmentation and border detection of melanoma lesions using convolutional neural network and SVM," Computational Intelligence: Theories, Applications and Future Directions, pp 97-108, 2018.

[12] J. Li, K. Sarma, K. Chung Ho, A. Gertych, B. Knudsen, C. Arnold, "A Multi-scale U-Net for Semantic Segmentation of Histological Images from Radical Prostatectomies," AMIA Annu Symp Proc. 2018; 2017 1140âĂŞ1148. PMID: 29854182, 2017.

[13] J. Long, E. Shelhamer, T. Darrell, "Fully convolutional networks for semantic segmentation," In: IEEE Conference on Computer Vision and Pattern Recognition (CVPR). IEEE, p.3431-40, 2015.

[14] L-C. Chen, G. Papandreou, I. Kokkinos, K. Murphy, AL. Yuille, "DeepLab: Semantic Image Segmentation with Deep Convolutional Nets, Atrous Convolution, and Fully Connected CRFs," IEEE Trans Pattern Anal MachIntell, 1-1, 2017.

[15] O. Ronneberger, P. Fischer, and T. Brox, "U-net: Convolutional networks for biomedical image segmentation," in MICCAI, 2015.

[16] R. Friedman, D. Rigel, A. Kopf, "Early detection of malignant melanoma: the role of physician examination and self-examination of the skin," CA-Cancer J Clin, 35:130âĂŞ51, 1985.

[17] D. Pathak, P. Krahenbuhl, T. Darrell, "Constrained convolutional neural networks for weakly supervised segmentation," in ICCV, 2015.

[18] N. Codella, D. Gutman, M. Emre Celebi, B. Helba, M. Marchetti, S. Dusza, A. Kalloo, K. Liopyris, N. Mishra, H. Kittler, A. Halpern: "Skin Lesion Analysis Toward Melanoma Detection: A Challenge at the 2017 International Symposium on Biomedical Imaging (ISBI), Hosted by the International Skin Imaging Collaboration (ISIC),' arXiv:1710.05006, 2017.

[19] P. Tschandl, C. Rosendahl, H. Kittler, "The HAM10000 dataset, a large collection of multi-source dermatoscopic images of common pigmented skin lesions," Sci. Data 5, 180161 doi:10.1038/sdata.2018.161, 2018.

[20] W. Shimoda and K. Yanai, "Distinct class-specific saliency maps for weakly supervised semantic segmentation," in ECCV, 2016.

[21] K. Hua,"A comparison of edge detection methods for segmentation of skin lesions in mobile-phone-quality images," Bachelor of Science thesis, Edith Cowan University, 2007. 
[22] N. Kanopoulos, N. Vasanthavada, R.L. Baker, "Design of an Image Edge Detection Filter using the Sobel Operator," Journal of Solid State Circuits," IEEE, vol. 23, Issue: 2, pp. 358-367, 1988.

[23] H. Zimmermann, "Fuzzy set theory and its applications," Kluwer Academic Publishers, second edition, 1991.

[24] D. Rigel, R. Friedman, A. Kopf, D. Polsky, "ABCDE-an evolving concept in the early detection of melanoma," Arch Dermatol, 141:1032-4, 2005.

[25] C. Lee, Y. Kuo, P. Yu, "Weighted fuzzy mean filters for image processing," Fuzzy Sets Syst., no. 89, pp. 157-180, 1997.

[26] Z. Zhang, W. Stoecker, R. Moss, "Border detection on digitized skin tumor images," IEEE Trans Med Imaging, 19(11): 1128-1143. doi:10.1109/42.896789, 2000 .

[27] M. Celebi, Y. Aslandogan, P. Bergstresser, "Unsupervised border detection of skin lesion images," in Int. Conf. Information Technology: Coding and Computing, 2005, vol. 2, pp. 123-128, 2005.

[28] Q. Abbas, I. Garcia, M. Rashid, "Automatic skin tumor border detection for digital dermoscopy using a novel digital image analysis scheme," British Journal of Biomedical Science 67, 177-183, 2010.

[29] S. Umbaugh, R. Moss, W. Stoecker, "An automatic color segmentation algorithm with application to identification of skin tumor borders," Computerized Medical Imaging and Graphics 16(3), 227-235, 1992.

[30] G. Hance, S. Umbaugh, R. Moss, W. Stoecker,"Unsupervised color image segmentation with application to skin tumor borders," IEEE Eng Med Biol., 15:104-111, 1996.

[31] R. Dice, "Measures of the Amount of Ecologic Association Between Species," Ecology. 26 (3): 297-302. doi:10.2307/1932409. JSTOR 1932409, 1945. 